\title{
The Use of Marigold Therapy for Podiatric Skin Conditions
}

\author{
by Robert A. Hadfield, BS ${ }^{1}$, Tracey C. Vlahovic, DPM ${ }^{2} \rrbracket$, M. Tariq Khan, PhD (Lond), BSc (Pod \\ Med), BSc (Hons), MChS, DFHom (Pod), FBAHChP PFLS ${ }^{3}$
}

The Foot \& Ankle Journal 1 (7): 1

Marigold therapy has been used for over 30 years in the United Kingdom and has been evaluated by numerous randomized double-blind placebo-controlled studies for various skin issues on the lower extremity. Various species of marigold are naturally anti-viral, keratolytic, and anti-inflammatory when applied topically to the affected area. Marigold therapy offers a non-invasive and gentle treatment for difficult to treat plantar verruca, painful hyperkeratotic lesions, and inflamed bursa secondary to hallux abducto valgus.

Key words: Phytotherapy, Marigold, verrucae, foot ulcer, Tagetes species, Calendula species, hyperkeratotic lesions, bunion, bursitis

Accepted: May $2008 \quad$ Published: July 2008

This is an Open Access article distributed under the terms of the Creative Commons Attribution License. It permits unrestricted use, distribution, and reproduction in any medium, provided the original work is properly cited. (The Foot \& Ankle Journal (www.faoj.org)

$\mathbf{T}$ Wwo marigold species, Tagetes and Calendula, have been used for centuries as herbal remedies for various ailments. (Figs.1ab) Phytotherapy, or the use of plants for their medicinal properties, is the basis for most pharmaceutical products. In particular, the marigold has an interesting history as both a topical and an oral remedy.

Address correspondence to: Tracey C. Vlahovic, DPM Associate Professor, Temple University School of Podiatric Medicine, Philadelphia, Pa. 19107 email: traceyv@tample.edu

${ }^{1}$ Fourth year student, Temple University School of Podiatric Medicine, Philadelphia, Pa. 19107.

${ }^{2}$ Associate Professor, Temple University School of Podiatric Medicine, Philadelphia, Pa. 19107.

${ }^{3}$ Deputy Director of Moeopathic Podiatry, The Marigold Clinic, Royal London Homeopathic Hospital, London, UK.
Marigold therapy was first described in the podiatric literature as a treatment for plantar hyperkeratotic lesions in $1980 .^{1} \quad$ The Tagetes species of marigold has been found to be strongly keratolytic. The keratolytic and anti-inflammatory properties of Tagetes in the treatment of verrucae and hyperkeratotic lesions have been well documented in the literature..$^{2-8}$ Tagetes has also been described as a treatment for carbuncles and eye infection in India as well as for joint pain and muscular spasms in Brazil and Mexico.,10 In addition to being a treatment for verruca, this species has also been used in the treatment of allergic contact dermatitis as well as radiation dermatitis associated with breast cancer therapy. $^{11,12}$ 

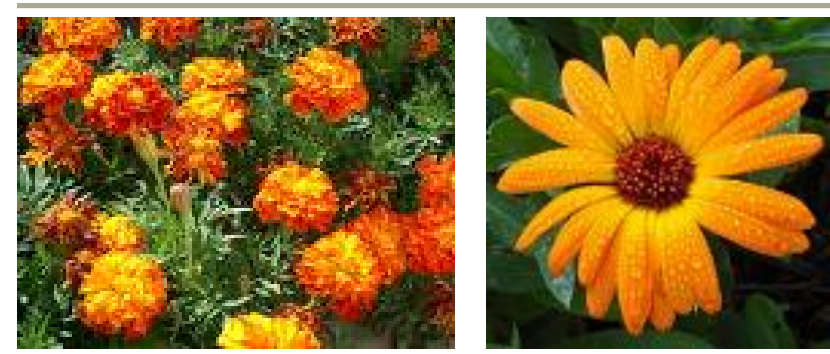

Figures 1ab The Tagetes (a) and Calendula (b) marigold species.

In contrast, the English pot Marigold (Calendula species) has been described in the treatment of cuts, wounds and ulcerations as early as $1838 .{ }^{13}$ More recently, anti-tumor and anti-oxidant properties of Calendula species have been established and utilized in researching treatments for various cancers. ${ }^{14,15}$ This species has also been found to have hepatocytoprotective properties in the treatment of $\mathrm{CCI}_{4}$ poisoning as well as antimicrobial properties. ${ }^{16-18}$ In recent years, investigators outside the United States have studied the properties of the Calendula species in healing diabetic foot ulcerations. ${ }^{19,20}$

This article will review the literature for the use of Tagetes and Calendula species on the most common podiatric conditions treated and will show their usefulness as an effective and non-invasive therapy. The chemicals found in the multiple Tagetes species described will demonstrate keratolytic, anti-viral, and anti-inflammatory properties. The use of the Calendula species of marigold will be described, including a case study demonstrating its use in non-healing ulcerations.

\section{Hyperkeratotic Lesions}

Painful hyperkeratotic lesions, arising from both mechanical stressors and boney deformity, are extremely common podiatric conditions. Treatments range from conservative debridement, change of shoe gear, orthotics, and topical keratolytics to surgical correction of the underlying deformity.

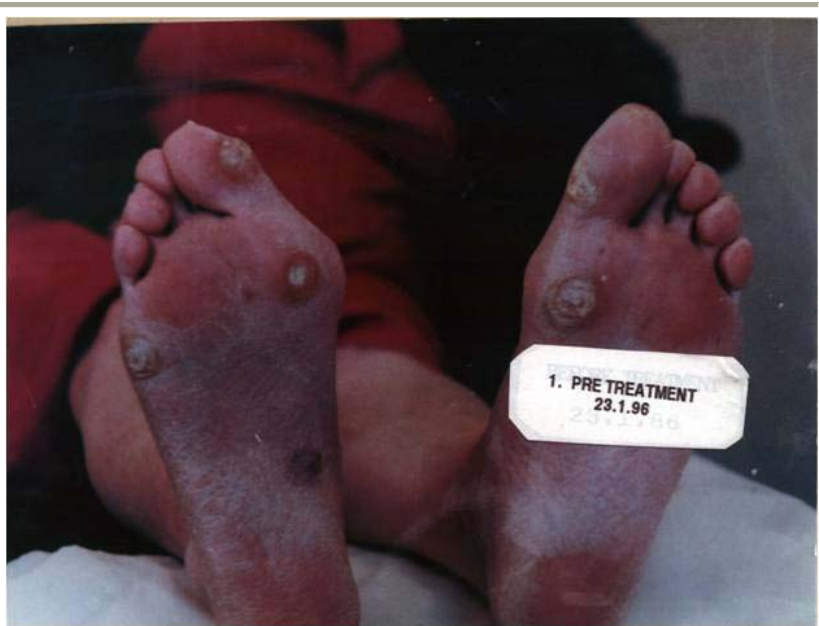

Figure 2 Painful plantar hyperkeratotic lesions prior to marigold therapy.

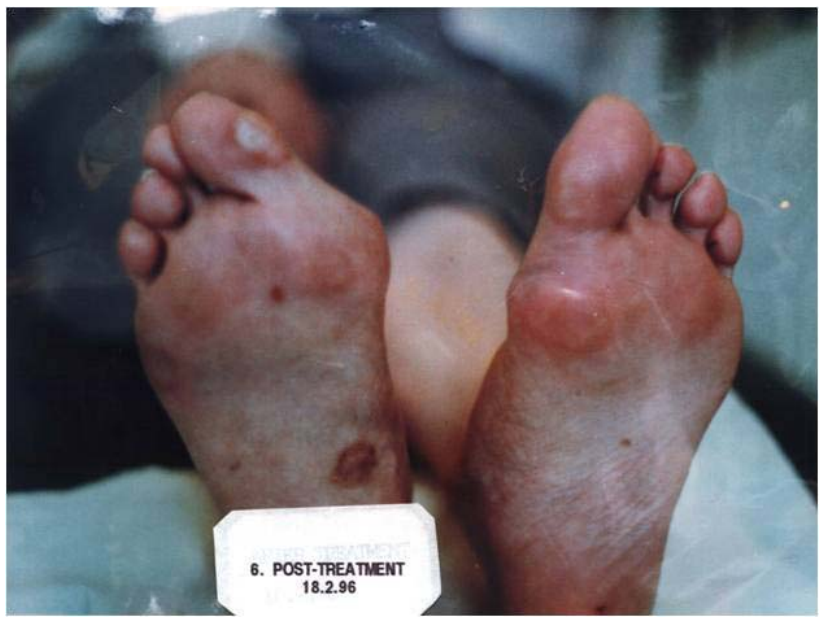

Figure 3 Clearance of lesions after four treatments; one month after initial therapy.

A marigold based paste has been used successfully as topical therapy for these lesions for many years in the United Kingdom. (Figs. 2, 3)

Davidson reported M. Taufiq Khan's work with the Tagetes species and its ability to inhibit cell activity in the stratum corneum. ${ }^{1} \quad$ A chemical analysis of Tagetes isolated tagetone, d-limonene, acimene, linalyl-acetate, linalol 9.8\%, and other terpenes. ${ }^{21}$ Tagetone acts as a catalyst to inhibit the rapid production and transmission of keratinocytes. ${ }^{22,23}$ 


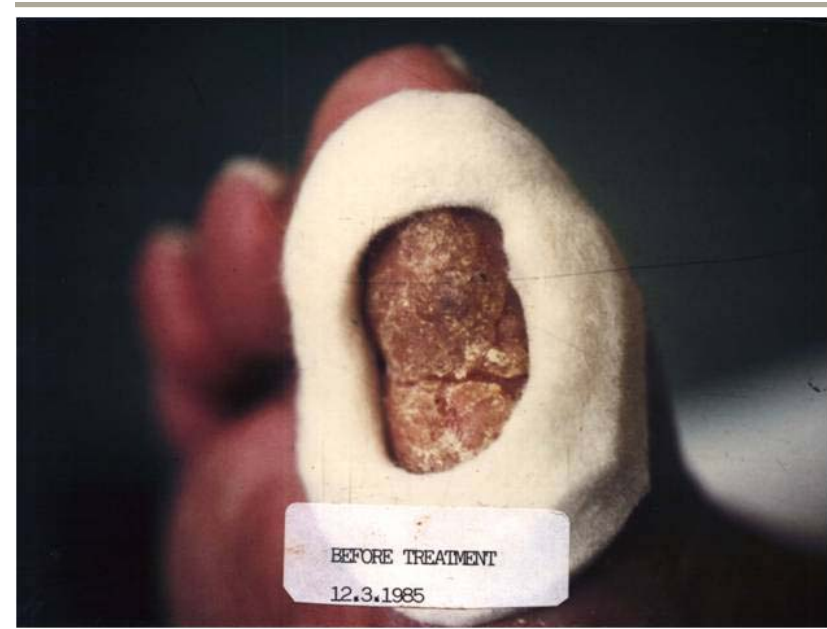

Figure 4 Recalcitrant plantar verruca prior to marigold therapy.

Tagetes' effect on podiatric skin, bone, and nail conditions was further described by Khan and White followed by numerous randomized, double-blind placebo controlled studies on those conditions. $^{24}$

In 1996, Tagetes erecta was used for a double blind placebo controlled study for thirty patients with painful plantar hyperkeratotic lesions. ${ }^{4} \quad$ They were separated into three groups: marigold therapy with an aperture pad, marigold therapy without an aperture pad, and placebo marigold therapy with aperture pad. The placebo paste was formulated to look and feel identical to the active paste but with no active chemical ingredients. In the semi-compressed felt aperture pad, a paste of fresh plant combined with isopropyl alcohol was applied by the podiatric practitioner once a week for four weeks over the painful lesions. The patients wore the poultice dressing for a week and returned weekly for lesion debridement by the practitioner. At the end of the treatment period, patients received either active or placebo tincture and ointment to use on the test area at home for four weeks.

The active paste group with a pad showed a significant decrease in hyperkeratotic lesion width, length, and pain when compared to the placebo group.

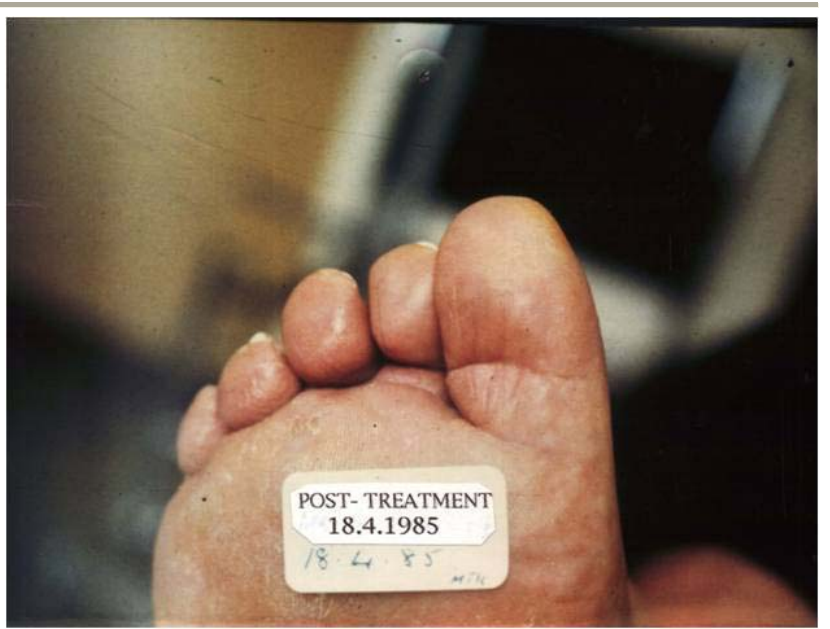

Figure 5 Clearance of the lesion after four treatments.

The active paste group with pad versus the active paste group without pad also had a significant difference in width, length and pain showing that the aperture pad had helped to offload the lesions as well as compartmentalize the marigold paste to the affected area. Reducing the trauma at the painful hyperkeratosis site is believed to decrease cytokine production thus decreasing kertinocyte production. This coupled with the natural keratolytic property of Tagetes (containing tagetone among other chemicals) proved to be a promising therapy, which could be used prior to custom molded orthotic therapy or as an alternative to surgical correction.

The keratolytic property of Tagetes was further explored in several published case studies. ${ }^{5,13,25}$ Patients receiving a similar therapy as previously described reported pain relief after 48 hours of the first dressing application. Interestingly, the patients who used the home therapy and orthotic control after the initial eight week period had no recurrence of the lesions after one year.

\section{Plantar Verrucae}

Plantar verrucae cause patients to seek treatment when the lesions become painful or do not resolve on their own. 


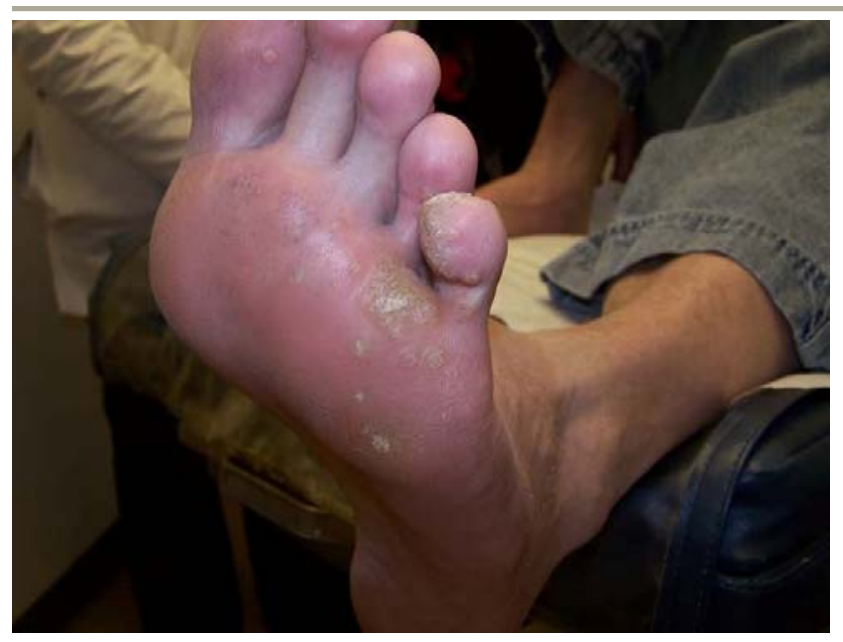

Figure 6 Immunocompromised patient with mosaic plantar verruca prior to therapy.

Treatments range from topical to surgical and are typically painful. Marigold therapy can also be added to the many topical treatments for verrucae. (Figs. 4,5) Forty patients were randomly placed into one of four groups; active, placebo, active with pad, and pad only with no paste. $^{26}$ Patients were treated twice a week for two weeks and then used home therapy consisting of the tincture and ointment for four weeks. The lesion surface area pre-treatment and posttreatment was analyzed with a wound mapping system. Results showed that the active group had a significant difference in appearance, pain, and size compared with the placebo group.

Additionally, the proposed antiviral property of Thuja occidentalis, a member of the conifer tree family, was studied using thirty randomly selected patients. ${ }^{27}$ Patients were chosen with lesions older than eighteen months. An extract containing $T$. occidentalis was applied daily for three weeks and followed for six months after initial treatment. Ninety percent of patients had resolution of their lesion after one month from the initial treatment. At six months, the same number of patients had no recurrence. After the assessment, a double blind placebo controlled study was done. Results showed $80 \%$ of the active group had resolution while the placebo group had just $33 \%$ resolution. $^{27}$

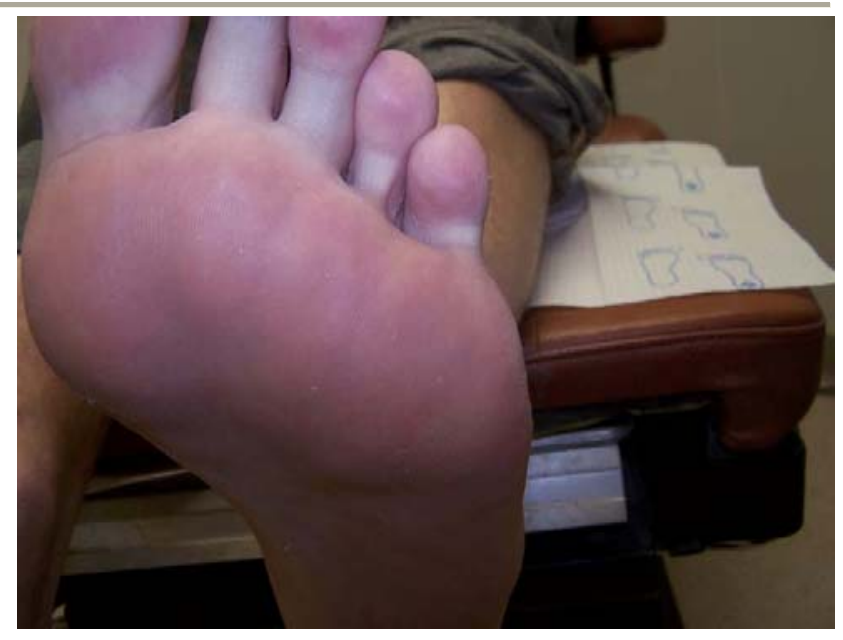

Figure 7 Clearance of mosaic verruca after four treatments.

A combination of the Tagetes and Thuja into a paste has been used extensively since the separate controlled studies were completed. A small case study showed the usefulness of the paste in immunocompromised patients with mosaic type verrucae. ${ }^{28}$ All of the patients were HIV positive with detectable viral loads and verrucae that did not respond to conventional treatment. Four applications of the combination paste eradicated the verrucae in two of the three patients. (Figs. 6,7) Although further research is warranted in this population, it is a hopeful outcome for a noninvasive treatment.

\section{Soft Tissue Inflammation Associated with Hallux Valgus}

The tissue underlying the prominent medial eminence seen in hallux valgus can become inflamed and be the source of a patient's discomfort. This bursitis is often treated with injections, padding, and shoe gear change. For patients that do not want surgery to correct the underlying boney deformity, marigold offers a gentle alternative to decrease inflammation and pain associated with hallux valgus. (Figs. 8,9) There is strong evidence that a particular species of Tagetes exerts anti-inflammatory action towards acute and chronic conditions. ${ }^{29}$ 


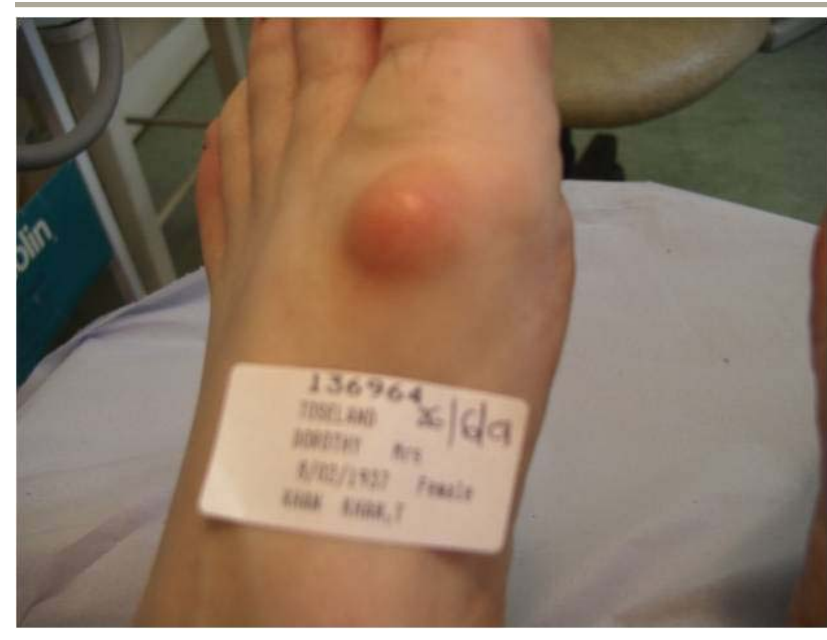

Figure 8 Patient with significant bursitis pain over bunion prior to marigold therapy.

A randomized double-blind placebo controlled study utilized sixty patients with either bilateral or unilateral inflammation was performed. ${ }^{30}$ Twenty patients with bilateral inflammation were randomly placed into one of two groups: active paste with aperture pad and placebo paste with protective pad. Forty patients with unilateral bursitis were randomly placed into similar groups with an identical treatment plan. All patients followed the paste and pad therapy with either an active or placebo home therapy consisting of tincture and ointment spray to use on the area daily. Soft tissue swelling at the medial eminence of the bunion was assessed using calipers pre and post treatment. Patients in both of then active groups had complete relief of pain after eight weeks and a 35\% reduction in soft tissue swelling at the bursitis site. In the placebo group showed minor reduction in pain which was most likely due to the presence of the aperture pad. However, their original symptoms returned at a week 4 when the patients started the placebo home therapy.

Marigold's ability to reduce bursitis inflammation was further studied in a group of 45 patients with unilateral pain. ${ }^{22}$ Patients were randomly placed into five groups: six groups with active marigold paste (each group had various extracts in organic solvents) and aperture pad and three groups with placebo without aperture pad.

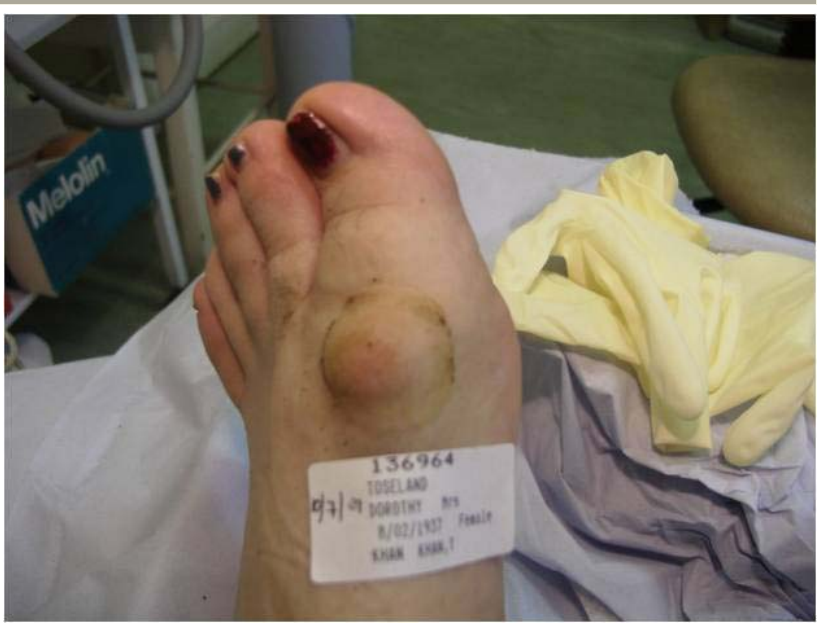

Figure 9 Decrease of erythema and inflammation at bunion site after therapy.

After a similar treatment plan described in the previous study, Group A (active paste in ethanolic extract with pad) had 100\% in level of pain (using the visual analogue pain scale) while Group G had $30 \%$ reduction in their level of pain.

Both of these studies support the combined use of marigold therapy with a protective aperture pad for patients with painful medial eminence bursitis who are not surgical candidates or do not wish to undergo surgical correction.

\section{Superficial Ulceration}

Khan states, in his 1982 publication in World Medicine on the uses of various species of marigolds, that Calendula species promote healthy granulation tissue. ${ }^{13}$ There are currently clinical projects underway at the School of Pharmacy at the University of London on these properties of Calendula. There was a single reported case of use in a diabetic ulcer, by Khan in the United Kingdom, using the formulation that was utilized in the following case study.

A larger study, by Duran, et al., was published in Serbia in 2005, using Calendula extract on 34 venous stasis ulcerations. ${ }^{20}$ 


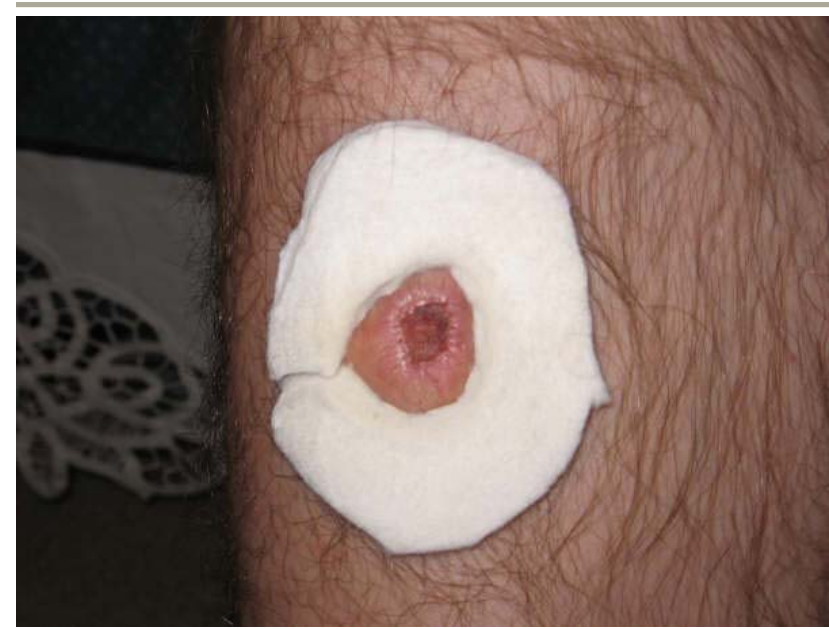

Figure 10 Anterior leg ulcer on intital presentation prior to marigold therapy.

They reported a statistically significant difference in reduction of total wound area compared with the control $(\mathrm{p}<0.05)$, showing an overall decrease of $41.71 \%$ in the experimental group compared with $14.52 \%$ in the control group. They conclude that application of Calendula extract significantly increases epithelization in chronic venous ulcerations.

This study by Duran, et al., lends scientific credence to the use of Calendula extract as a treatment to decrease the healing time of chronic ulcerations. However, randomized control studies have to yet to be completed.

\section{Case Study}

A thirty year old Caucasian male presented with concern of an ulcer on the anterior right leg. The superficial wound was over the site of an external fixator pin tract scar, which had occurred fifteen years earlier. The injury was a result of a blunt blow on a coffee table, shearing off the hypertrophic scar. The patient reported bleeding globally across the lesion at the time of injury. He denied a personal or family history of family diabetes, hypertension, coagulopathy and peripheral vascular disease.

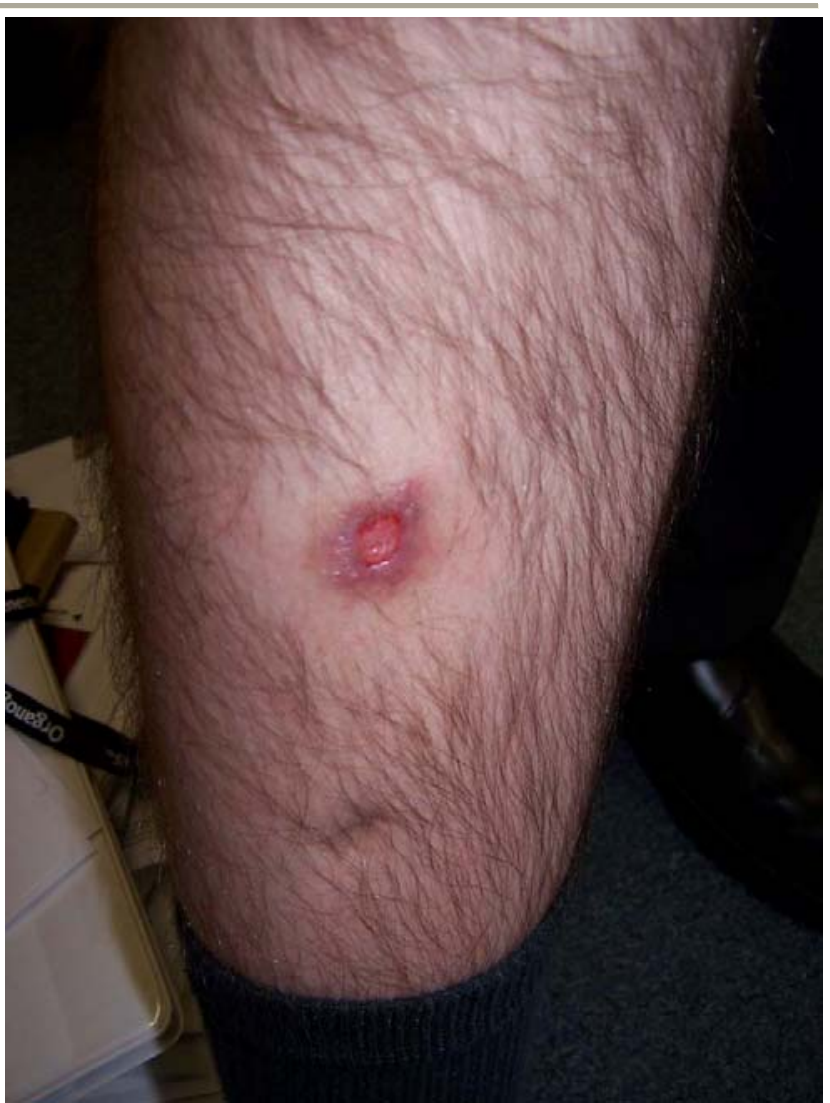

Figure 11 The lesion with decreased erythema and increased granulation tissue 48 hours after therapy.

Treatment initiated by the patient consisted of triple antibiotic ointment and a bandage. The patient reported that approximately one week after the injury, in the course of cleansing the wound, eschar that had previously formed selfdebrided, and the wound appeared as it did on the day it was sustained. The same treatment was resumed, with a similar course of routine healing. Two weeks following the injury, a similar loss of eschar was duplicated. The second event led the patient to seek treatment.

On initial examination, the patient had a $2.5 \mathrm{~cm} \mathrm{x}$ $2 \mathrm{~cm} \times 0.5 \mathrm{~cm}$ circular lesion over the crest of the anterior tibia in the central one third of the leg. The lesion had a mixed granular and fibrotic base, with mild surrounding erythema and no edema. (Fig. 10). 


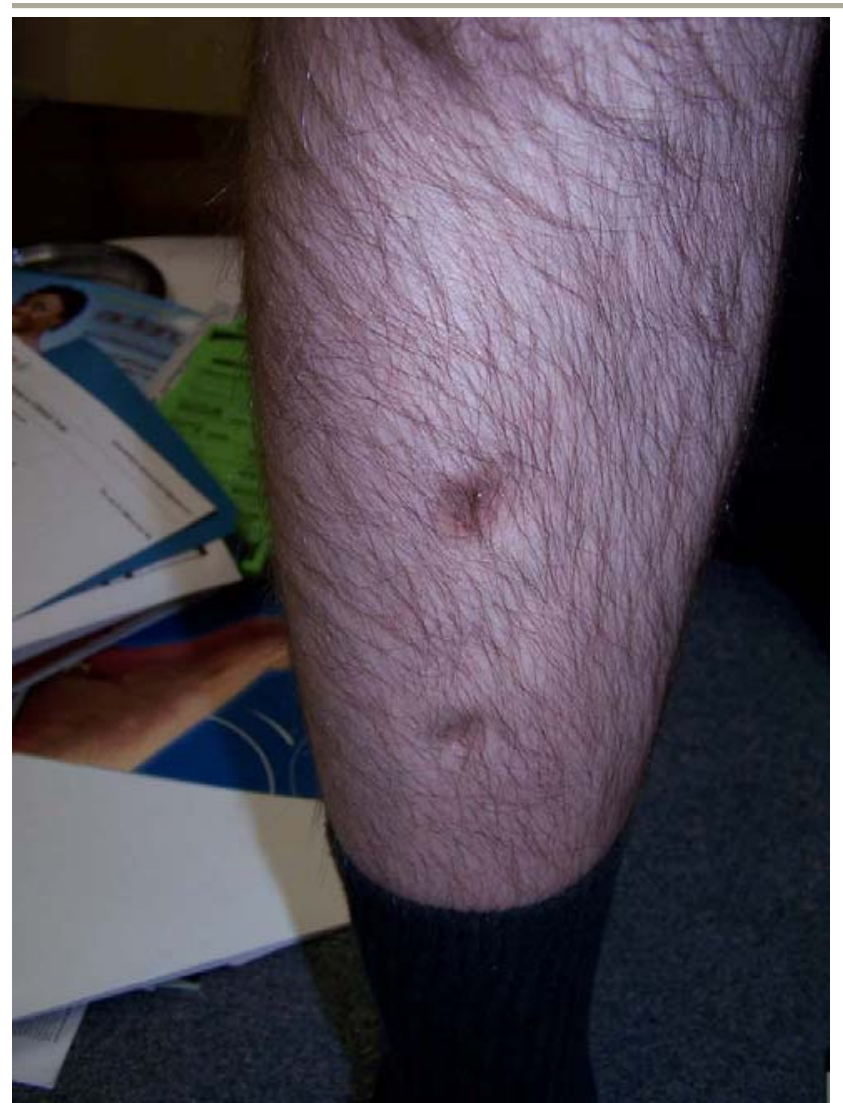

Figure 12 The resolved lesion.

No undermining was present, and there was no maceration of the wound edges. There was no purulence or malodor. The scars from the other pin tract sites were also examined and were labeled hypertrophic.

The patient received a regimen of marigold therapy for the wound. The medication used was the HTS 087 Tincture and Ointment. (Marigold Footcare Ltd, UK) The therapy regimen consisted of a combination of the tincture and ointment placed in an aperture pad which was then covered with medical tape and gauze over the lesion for three consecutive days. The same regimen was followed every other day after the initial three day period for two weeks.

The patient related no pain or discomfort for the duration of the treatment. Reduction in wound size (approximately 25\%) was noted 48 hours after marigold therapy was initiated. (Fig. 11)
Eschar information with a decrease in wound size was noted on subsequent dressing changes for six days afterwards. The directions for application were followed until epithelization was noted, which was accomplished in eight days. Complete resolution was obtained in approximately four weeks. (Fig. 12)

The complex nature of this wound, having formed over a pre-existing scar and in a traditionally difficult-to-heal area anatomically, caused a delay in wound healing despite the patient's uncomplicated medical history. The wound's recalcitrance in healing warranted additional therapy than just antibiotic ointment and patience.

\section{Discussion}

It should be noted that over the counter and/or health food store creams and preparations of marigold do not have the same effect as the previously discussed studies show. The extracts used for the controlled and case studies were researched and developed by M Taufiq Khan and M Tariq Khan over thirty years. They developed specific extracts that are directly applied by the podiatric physician to the patient: an anti-viral paste (for verruca), an anti-inflammatory paste (for bursitis and tendonitis), a keratolytic paste (for hyperkeratosis), and an anti-fungal paste (for nails). The patients then continue with home therapy that consists of tinctures and ointments with the same properties. These products were only recently introduced to the United States. The second author was the first United States podiatric physician to become certified in the use of the marigold products from Marigold Footcare, Ltd., and the Royal London Homeopathic Hospital, London, UK and will be able to certify (in conjunction with the Hospital) other podiatric physicians in the future. In order to ensure appropriate use of the extracts, certification will only be available to podiatric physicians and is required for both the usage and purchasing of the products. Further research will continue into using the extracts on genodermatoses, onychomycosis, and other podiatric conditions. 


\section{Conclusion}

The superficial ulceration case study and immunocompromised patients with verruca case study combined with studies performed outside of the United States show the promise of the continued investigation of marigold therapy as a treatment for various podiatric conditions. Marigold therapy has consistently been shown to provide gentle, non-invasive treatment that allows patients a painless alternative treatment.

\section{References}

1. Davidson L., "Marigold rediscovered: a cure for callosities," Therapy. Pg 1, 1980.

2. Khan M., et al., "Homeopathic treatment of common foot conditions," British Journal of Dermatology Nursing. 1: 20 23, 2003.

3. Khan M., et al., "Marigold Treatment of foot conditions," Dermatology in Practice. 10 (3): 28-30, 2002. 4. Khan M., et al.,"Podiatric Treatment of hyperkeratotic plantar lesions with marigold Tagetes erecta," Phytotherapy Research. 10: 211-14, 1996.

5. Khan, et al., "Treatment of plantar hyperkeratosis with homeopathic podiatry," Podiatry Now. Dec. 502-505, 2000. 6. Talhouk R., et al., "Anti-inflammatory bioactivities in plant extracts," J Med Food. 10 (1): 1-10, Mar 2007.

7. Herold A., et al., "Antioxidant Properties of some hydroalcoholic plant extracts with anti-inflammatory activity," Roum Arch Microbiol Immunol. 62(3-4): 217-227, 2003.

8. Fuchs S., et al., "Protective effects of different marigold (Calendula officinalis L.) and rosemary cream preparations against sodium-lauryl-sulfate-induced irritant contact dermatitis, “ Skin Pharmacol Physiol. May 20, 2005.

9. Hernandez F. "Historia de las plantas de nueve Espana.” Mexico Irmenta Universitaria. 1: 90-91, 1942. 10. Caius J. "The Medical and Poisonous Compsoitae of India, "J Bombay Natural History Society. 41: 90-91, 1940. 11. Pommier P., et al., "Phase III randomized trial of Calendula officinalis compared with trolamine for the prevention of acute dermatitis during irradiation for breast cancer, "J Clin Onco.l 22 (8) ; 1447-1453, Apr 15, 2004. 12. Pirker C., et al., "Cross-reactivity with Tagetes in Arnica contact eczema," Contact Dermatitis. 26 (4): 217-219, Apr 1992.

13. Khan M. "Why marigolds can be a corny treatment: A pilot study of the effects of marigold in the treatment of painful corns," World Medicine. 42-43, Feb 1982.
14. Jimenez-Medina E., et al., "A new extract of the plant Calendula officinalis produces a dual in vitro effect: cytotoxic anti-tumor and activity and lymphocyte activation," BMC Cancer. 6: 119, 2006.

15. Wang M., et al., "Antioxidant activity, mutagenicity/anti-mutagenicity, and clastogenicity of lutein from marigold flowers," Food Chem Toxicol Epub Sp; 44(9) : 1522-1529, Apr 25, 2006.

16. Barajas-Farias L., et al., "A dual and opposite effect of Calendula officinalis flower extract: chemoprotector and promoter in a rat hepatocarcinogenesis model" Planta Med. 72(3) 217-221, Feb 2006.

17. Rusu M., et al., "The hepatoprotective action of ten herbal extracts in $\mathrm{CCl}_{4}$ intoxicated liver," Phytother Res. 19(9): 744-749, Sep 2005.

18. Radioza S., et al., "Antimicrobial activity of Calendula L. plants," Ukranian Journal of Microbiology. 21-25, 2007.

19. Khan M., et al., "Treatment of Diabetic foot ulcer with Homeopathic Podiatry," Poster Presentation, Symposium on Advances in Skin and Wound Care, Las Vegas, 2005.

20. Duran V., et al., "Results of the clinical examination of an ointment with marigold (Calendula officinalis) extract in the treatment of venous leg ulcers," Int J Tissue React. 27(3) ; 101-6, 2005.

21. Leung A Encyclopedia of Common Natural Ingredients, Used in Food, Drugs and Cosmetics. Academic Press, New York, 1980.

22. Khan M. "Phytochemical and biologic studies of Tagetes erecta and its clinical evaluation in the treatment of hallux abducto valgus and its associated condition bunion," $\mathrm{PhD}$ Thesis, Faculty Medicine, Centre for Pharmacognosy, School of Pharmacy, University of London, 1999.

23. Sharma A. "Tagetes genus," Wealth of India. Vol X, SP W p 109, 1961.

24. Khan M., et al., "Comparative clinical studies of Calendula offcinalis and Tagetes varieties of marigold," $35^{\text {th }}$ LMHH Congress, Sussex, 488-492, 1982.

25. Khan M., et al., "Clinical evaluation of Tagetes erecta in the treatment of parakeratosis," Phytotherapy Res. 10: 186188, 1996.

26. Khan M. Jnr. "Tagetes signata in the treatment of verruca pedis," J British Pod Med. 51: 118b, 1996.

27. Khan M., et al., "A double blind placebo study of topical Thuja occidentalis on verruca pedis in children and adults," JEADV. 1: S251, 1999.

28. Vlahovic T., et al., "The Use of Marigold Therapy on Mosaic Verrucae in the HIV population," Presented at AAD, San Antonio, TX 2008. JAAD. 58(2): AB34, 2008. 29. Kasahara Y., et al., "Effect of methanol extract from flower petals of Tagetes patula $L$. on acute and chronic inflammation model," Phytother Res. 16(3): 217-222, 2002. 30. Khan M. "The podiatric treatment of hallux abducto valgus and its associated condition bunion with Tagetes patula," J Pharm Pharmacol. 48: 768-770, 1996. 